\title{
HUBBLE SPACE TELESCOPE SECOND GENERATION INSTRUMENT SELECTION
}

\author{
E. J. WEILER \\ Hubble Space Telescope Program Scientist \\ Code EZ \\ NASA Headquarters \\ Washington, DC 20546 \\ USA
}

\begin{abstract}
The Hubble Space Telescope Second Generation Instrument Program is described. The original instrument selection process in 1985 is discussed as well as the NASA plan to make a final selection of an infrarcd instrument in late 1988.
\end{abstract}

An Announcement of Opportunity (AO) for Hubble Space Telescope (HST) Second Generation Instruments was issued by NASA in October, 1984. NASA received eight proposals as a result of this AO. A sixteen member scientific peer review team evaluated these proposals and provided NASA with a summary of each proposal's strengths and weaknesses. They also provided unsolicited advice on the relative priorities of various classes of instruments. Although the team was primarily composed of ultraviolet and visible astronomy experts, they rated an infrared camera/spectrometer as the number one priority. An ultraviolet/visible spectrograph was rated second priority.

NASA considered the peer panel's advice and selected three proposals for Phase A/B studies. This decision was announced in December, 1985. Since the peer panel could not provide clear discriminators between the two top infrared instruments, NASA decided to select both for definition studies with the intent of carrying only one into development. The selected infrared instruments were the Near-Infrared Camera and Multi-Object Spectrometer (NICMOS), proposed by Dr. Rodger Thompson of the Unversity of Arizona, and the Hubble Imaging Michelson Spectrometer (HIMS), proposed by Dr. Donald Hall of the University of Hawaii. The third instrument selected was the Space Telescope Imaging Spectrograph (STIS), proposed by Dr. Bruce Woodgate of the Goddard Space Flight Center.

Although NASA's original plan was to carry both infrared instruments through full Phase A and Phase B studies, it was decided in late 1987 to initiate an early selection process. This decision was made both to save money and because the respective Principal Investigators (P.I.'s) felt that enough progress had been made to enable an early selection. A plan to enable an early selection was developed by NASA and the P.I.'s during the early part of 1988. This plan calls for final proposals from both the NICMOS and HIMS teams by August 26, 1988. During September, 1988, two travelling peer review teams will visit contractor and home institution sites to conduct indepth technical 
reviews of detector technology and instrument cryogenics and mechanisms. In addition to these specific technical reviews, the Goddard Space Flight Center will conduct a broad technical review of both instruments. The overall proposals and the results of the aforementioned reviews will be evaluated by a summary peer review panel in early November, 1988. Selection criteria will be those listed in the original A.O. (e.g., overall scientific merit, technical merit, etc.). NASA will announce a final selection in early December, 1988. The selected instrument will then be funded to complete its Phase B study and then proceed into instrument development.

The current NASA plan calls for the development of the infrared instrument first. This is based on the fact that there is no infrared capability in the original complement of HST instruments and on the strong scientific recommendations of the original peer review team. Under this plan, the infrared instrument would be launch ready in August, 1994. The STIS would follow with a launch readiness about 1-2 years later. This plan could be altered in the event that there are early problems with one or both spectrographs in the original complement of HST instruments. In other words, the STIS could precede the infrared instrument if such a decision were made shortly after the launch of the HST. 